\title{
De crisis a crisis: la invención de la desocupación en la Argentina
}

$$
\text { por }
$$

Claudia Daniel

UBA-IDES-CONICET

\begin{abstract}
El artículo estudia el proceso histórico de configuración de la desocupación como problema social y estadístico en la Argentina de entreguerras, articulando tres ejes o dimensiones: la vinculación entre instrumentos estadísticos y formas de configuración estatal; la circulación internacional de saberes en torno a la cuestión del trabajo; el papel de las crisis (1914-17 y 1930) en la generación de representaciones estadísticas estables sobre la desocupación en el país.
\end{abstract}

Palabras Clave: Desocupación; Estadísticas sociales; Departamento Nacional del Trabajo; Crisis; Argentina; Entreguerras.

En 1995, con los efectos de la crisis del Tequila en la Argentina, el tema de la desocupación se instalaba definitivamente en la agenda política. Además de ser situaciones experimentadas por una parte significativa de la sociedad, los términos desempleo, subempleo, precariedad e inestabilidad laboral circularon en el espacio público definiendo tanto el contenido como los contornos de la cuestión social durante el orden neoliberal. En aquel año, la tasa de desocupación alcanzó niveles inéditos para el país. El órgano oficial de estadísticas presentaba públicamente el índice del desempleo, sintetizando en un frío cálculo el padecimiento colectivo de los ciudadanos alcanzados por los estragos sociales que traía aparejada la aplicación de reformas neoliberales en el país. La desocupación configuraría además una identidad social, la del movimiento político que, llevando la política a las calles, brindaría un carácter particular a la nueva cuestión social en la Argentina de fines de siglo XX. 
Si entendemos, junto al sociólogo francés Robert Castel (1997), a la cuestión social como la forma en que una sociedad experimenta el riesgo de su propia fractura, en la Argentina de los años '90 esa aporía fue interrogada públicamente por medio de la retórica de los números. La estadística se estableció como la herramienta válida de lectura del problema, mientras constituía la forma de darle entidad y visibilidad pública al mismo tiempo. En la puesta en locución de la cuestión social, los números del desempleo ganaron claramente protagonismo (en los debates políticos, académicos e incluso televisivos) y esa fuente, mezcla de conocimiento técnico, precisión y certeza, a los ojos de una sociedad impávida por el derrumbe de las ilusiones de bienestar que había generado inicialmente el modelo económico neoliberal, quedó poco a poco a merced de un debate público que se fue haciendo más amplio y excedió el círculo estrecho de especialistas. Con las tasas de desocupación como epicentro se jugaron las primeras batallas contra el discurso neoliberal dominante que había redefinido los límites y las funciones del Estado. Refiriéndose a ellas, el entonces Ministro de Economía Domingo Cavallo denunció como «mentirosos» los índices del Instituto Nacional de Estadísticas y Censos por no reflejar la «salud» de la economía bajo el patrón de la convertibilidad ${ }^{1}$. Pese a los embates contra su credibilidad, a cifra conservaba la confianza social general ganada por un organismo estatal que cultivó durante largos años los pilares de su autoridad social ${ }^{2}$. Otros economistas de renombre se sumaron al cuestionamiento de la fiabilidad de las estadísticas oficiales (sobre la creación de puestos de trabajo, la producción industrial y el $\mathrm{PBI}$ ), nutriendo una crisis de credibilidad que hoy en día, en un contexto económico, social, político, e incluso discursivo, totalmente diferente sigue su «proceso de descomposición», adoptando nuevos ribetes y a la espera de respuestas políticas.

Por otra parte, diversos autore ${ }^{3}$ han demostrado que la historia de los métodos estadísticos y de sus formalizaciones matemáticas está entremezclada, de manera compleja, con aquella de los objetos que son puestos en la agenda de las políticas sociales. En los países occidentales modernos, la

${ }^{1}$ Bajo el predominio social, político y cultural del paradigma neoliberal, con su discurso apologético del achicamiento del Estado y el auge privatista, se llegó a barajar oficialmente la opción de disolver el Instituto Nacional de Estadística y Censos (INDEC) y contratar consultoras privadas para que elaboraran las estadísticas públicas. A su turno, los principales referentes políticos del neolibreralismo, Ronald Reagan y Margareth Thatcher, habían reducido los fondos de las estadísticas oficiales en sus respectivos países en nombre del repliegue de la dirección estatal de la economía.

${ }^{2}$ Respecto del proceso histórico de construcción de la autoridad social de las estadísticas oficiales en Argentina, ver: Daniel, 2010.

3 Desrosières, 1996. Davidson, 2004. Topalov, 1994, entre otros. 
definición de un problema social, al menos del siglo XIX en adelante, quedó indisolublemente ligada a la categorización de poblaciones realizada por medio del lenguaje cuantitativo. Las estadísticas contribuyen a producir sus propios objetos configurando los hechos que están orientadas a medir. Las formas que las estadísticas crean, promueven una definición de los problemas en la agenda política. Visto desde otro ángulo, como propone Desrosières, en los modos de tratamiento de las cuestiones sociales, es decir, en el seno de las políticas públicas, es posible encontrar, de manera articulada, la configuración de representaciones estadísticas, modos de pensar la sociedad y la economía, y formatos de acción pública ${ }^{4}$. Este artículo se inscribe en el orden de trabajos que tienen como horizonte la pregunta por el sentido en que la cuantificación y los indicadores estadísticos contribuyen a performar al mundo social, en configuraciones variadas y en agencias políticas que no son otra cosa que piezas de un mismo rompecabezas.

Desde este lugar, el artículo recupera una parte de la historia de la configuración de la desocupación como problema social y estadístico en la Argentina, teniendo en cuenta la experiencia de los países capitalistas avanzados que no sólo la precedió, sino que le dio un marco y un espejo donde mirarse. ¿Cuándo y cómo se estableció el interés y la legitimidad de una estadística nacional en este dominio? ¿Quienes contribuyeron en esa empresa, a la vez cognitiva y política, de colocar a la desocupación en la agenda de cuestiones socialmente problematizadas? ¿Sobre qué instrumentos y nociones se montó la configuración estadística de la desocupación en la Argentina de entreguerras?

En la propuesta de análisis se ponen intencionalmente a jugar dos momentos de crisis (1914-1917 y 1929-1930) porque identificamos en ellos escenarios generadores de demandas de conocimientos sobre el mundo social; demandas que, estando principalmente orientadas al Estado y a sus agencias burocráticas, provenían de un gran abanico de actores sociales. En los contextos percibidos como críticos de la etapa de entreguerras, las estadísticas empezaron a verse involucradas como canal de consagración pública de problemas ${ }^{5}$. En otros trabajos pudimos mostrar que estas coyunturas alimentaron el consenso social en torno de la necesidad de expandir los márgenes de la estadística oficial ${ }^{6}$. Aquí nos interesa tener en cuenta que si bien las situaciones percibidas como

${ }^{4}$ Desrosières, 2011: 76.

${ }^{5}$ En la primera mitad del siglo XX, la estadística poco a poco formó parte del grupo de los saberes validados socialmente a los que recurrieron a su turno los distintos actores sociales, tanto subalternos como predominantes, para construir la entidad y relevancia de una situación crítica en el espacio público, fortalecer sus posiciones y propuestas en el debate, e incidir en el rumbo de las políticas encaradas por el Estado.

${ }^{6}$ Daniel, 2010. 
críticas pueden impulsar demandas a los saberes sociales, éstas no necesariamente logran consolidarse públicamente ni promueven procesos de creación de nuevas formas estadísticas de pensar la realidad social.

En relación a la cuestión de la desocupación, la coyuntura crítica de los años 1913-1917 fue el terreno propicio para un primer planteamiento de la urgencia y la necesidad de poner en marcha un programa de medición del fenómeno de la desocupación. Sin embargo, factores de diversa índole (una disputa generacional interna a la elite burocrática especializada, un modelo predominante de pensar la economía, el Estado y la sociedad, las incipientes capacidades institucionales de una agencia estadística recientemente creada) bloquearon la generación de configuraciones estadísticas estables respecto del fenómeno social de la falta de trabajo, aunque ello no clausuró por completo la búsqueda de instrumentos sólidos para medir la desocupación, abriendo un camino sembrado de discusiones técnicas y políticas. Una nueva crisis (1930) revitalizaría el proceso creativo de invención estadística, consagrando otras herramientas y construyendo nuevas representaciones sobre la desocupación. Por tanto, parecería haber cierta concomitancia entre las crisis y los momentos claves en la construcción de representaciones estadísticas sobre la desocupación. Teniendo en cuenta la primera mitad del siglo XX, y observando la aguda crisis social que empañó el ocaso del mismo siglo, la invención de la categoría social y estadística de la desocupación parecería haber estado marcada por el ritmo de las depresiones cíclicas y las tensiones sociales.

El propósito general de este trabajo es adentrarnos en el terreno de la coconstrucción entre herramientas estadísticas y política social con el objetivo particular de desentrañar los procesos sociales, políticos y técnicos que jalonaron la configuración de representaciones estadísticas sobre la desocupación entre 1913-1914 y 1939.

\section{¿CUÁNTOS SON LOS DESOCUPADOS? El DESPERTAR DEL INTERÉS POR CONTAR}

En el transcurso del año 1913, la desocupación se instaló como un tema de interés y preocupación en la opinión pública. En la prensa -tanto burguesa como obrera- se difundían cifras de obreros sin trabajo, afirmándose que el número era excepcional, contribuyendo a generalizar la percepción de un exceso de oferta sobre la demanda de trabajo en la Capital Federal. Además de apreciaciones divergentes, circulaban explicaciones de todo tipo: el exceso de oferta sobre la demanda de trabajo se vinculaba con la caída de las actividades industriales por la crisis, pero también a una afluencia «anormal» de inmigrantes al territorio de la capital del país. La cuestión de la desocupación 
se construía públicamente como problema en reflejo de la situación que atravesaban algunos países europeos en vísperas de la Primera Guerra Mundial. El conflicto bélico implicaría para la Argentina trabas en su comercio exterior y la disminución de los flujos de capitales, factores que sumados a las malas cosechas de los años 1913-1914, desataron una crisis local con alzas en el costo de vida que duró hasta el año $1917^{7}$.

En el contexto convulsionado del período 1913-1917, se abrió un debate público respecto de la existencia «real» del fenómeno de la desocupación, su intensidad y sus alcances. Se recurría a la opinión de especialistas en la materia (como Marco Avellaneda, Augusto Bunge, Francisco Stach) que se expresaron en distinto tipo de consultas públicas. Las entidades representativas del sector obrero y patronal también se vieron involucradas en la polémica. Entonces, se encontraba en funcionamiento una agencia estatal dedicada a los temas relativos al trabajo, nacida azarosamente en 1907, pero oficializada en 1912 (ley orgánica 8.999). El Departamento Nacional del Trabajo (DNT) expresó el interés de cierto sector reformista del círculo de políticos liberales locales preocupado por la cuestión obrera ${ }^{8}$. Esa creación institucional seguía el modelo de las agencias gubernamentales organizadas en Francia, Inglaterra y Estados Unidos a fines del siglo XIX, con el propósito de observar, conocer y legislar el campo del trabajo ${ }^{9}$. La inquietud pública generalizada en torno a los niveles de desocupación interpelaba directamente a una de sus divisiones dedicada a producir estadísticas sociolaborales (en adelante, DE-DNT), a cargo del ingeniero Alejandro Bunge entre 1913 y 1915. Bajo la dirección de este ingeniero formado en Alemania y profesor de estadística en la Facultad de Ciencias Económicas de la Universidad de Buenos Aires (FCE-UBA) un plantel estrecho de funcionarios burocráticos (que no superó los diez empleados) llevaba adelante un programa de indagaciones cuantitativas y aplicaba encuestas y mediciones sobre el mundo del trabajo ${ }^{10}$.

${ }^{7}$ Gerchunoff y Llach, 1998: 68-100.

8 Zimmermann, 1995, Suriano, 2000; 2012.

9 Entre las décadas de 1890 y de 1910, un movimiento internacional se ocupó de las cuestiones del trabajo, desde aspectos tanto jurídico y administrativo como científicos. En 1891 fue creada en Francia una Oficina del Trabajo, a la que se integró la Satistique Generale de la France. Otros países institucionalizaron una serie de encuestas centradas en los trabajadores: Carroll Wright en los Estados Unidos, desde el Bureau of Labor Statistics, Engel en Alemania, Rowntree y Bowley en Inglaterra. En este último país la investigación social se transformó en parte de una rutina burocrática que fue integral al proceso de construcción, implementación y regulación de la política social. Al respecto, ver: Desrosières, 1999, Davidson, 1990; 2004, Rueschemeyer y Skocpol, 1996, Topalov, 1994.

${ }^{10}$ Los trabajos estadísticos del DNT fueron paralelos a los informes monográficos y descriptivos producidos por los inspectores laborales. Los especialistas estadísticos compartieron 
Cuando la desocupación se constituyó como un tema de relevancia social, el ingeniero Bunge propuso introducir en el tercer censo general de población proyectado para 1914 preguntas al respecto ${ }^{11}$. Su propuesta estaba socialmente respaldada en el vacío de datos sobre la ocupación que otros actores como los partidarios socialistas habían construido como demanda de recuento y clasificación al aparato estadístico nacional ${ }^{12}$. Sin embargo, su criterio fue desestimado por la comisión responsable del censo, conformada en esa ocasión por las figuras más importantes de la gesta fundacional de la estadística nacional durante el orden político conservador, los estadígrafos Alberto Martínez, Francisco Latzina y Eduardo Lahitte. La justificación de la comisión tripartita fue que preguntas de ese tipo «en momentos en que el país atravesaba por una perturbación económica que había dejado a muchas personas sin trabajo, habría fotografiado un estado social que no era el normal e inducido a equivocadas deducciones en un país de inmigración» ${ }^{13}$.

En torno a este censo se generó una controversia que marcó el pasaje de una generación a otra de especialistas de la estadística pública. Por un lado, la polémica fue el bautismo del ingeniero Bunge en la esfera pública en su rol de estadístico de renombre. Por el otro, fue la ocasión para que, utilizando como medio las revistas especializadas y las conferencias públicas, Bunge desacreditara la tarea de un consagrado en el ámbito especializado de la actividad como Martínez, director de la estadística municipal desde 1888, por carecer de método y bases científicas, disputándole el lugar central como voz autorizada en la materia.

con estos últimos el objetivo de generar un diagnóstico de la situación de la clase obrera en la Argentina. Sus resultados se difundían en los Boletines del Departamento Nacional del Trabajo y, a partir de 1918, también empezaron a aparecer en las Crónicas Mensuales del bureau laboral. Para una historia institucional sobre la DE-DNT ver: González Bollo, 1999; 2004.

${ }^{11}$ Boletín del Departamento Nacional del Trabajo, diciembre de 1913: 949-965.

${ }_{12}$ En opinión de los socialistas, el censo nacional resultaría más útil si recogía los datos sobre desocupación en todo el país, tal como se había hecho en Estados Unidos y Francia ( $\mathrm{La}$ Vanguardia, 30/7/1913: 1). Los socialistas exigían que el censo de población programado para junio de 1914 profundizara en la indagación de las condiciones de vida de la familia obrera, preguntando por la jornada de trabajo, los salarios, la periodicidad de la desocupación, si ella existía, pues consideraban que «para apreciar el grado de progreso de la República no basta conocer el crecimiento de su riqueza ganadera, ni el número de los habitantes que saben leer y escribir. Hay que conocer también las condiciones de vida y de habitación que a la mayoría de los habitantes les están reservadas» (La Vanguardia, 26/9/1913: 1). Especialistas en materia laboral, como Francisco Stach, denunciaban también la falta de información sobre aspectos importantes como salarios obreros, así como la carencia de uniformidad en otro tipo de registros vinculados a la cuestión obrera (Boletín del Museo Social Argentino, 1914, no 25-26: 49).

13 Tercer Censo Nacional, 1914, TI: 41. 
Pero más allá de las diferencias incluso ideológicas que los separaban, la forma misma en que era pensado el tema del trabajo desde el paradigma censal (Otero, 2006) resultaba reticente a aprehender la desocupación. Martínez era continuador de la forma tradicional en que el censo local capturaba el estatus ocupacional de las personas a través de la pregunta sobre «profesión, oficio o medio de vida habitual». Este enfoque entendía la ocupación como una característica del individuo (asimilable a la de sexo o edad), independientemente de que dicha habilidad estuviera siendo utilizada al momento del censo, es decir, más allá de que esa persona estuviera realizando una actividad laboral efectiva. Esta concepción asumía implícitamente cierta inmovilidad o fijeza de los sujetos en el mercado de trabajo. Sin embargo, la inestabilidad y la movilidad ocupacional eran características del mercado laboral argentino a principios de siglo XX, producto del tipo de trabajo (estacional o temporario) que ofrecía la estructura productiva, tanto en las áreas rurales (actividades de exportación) como en las ciudades (la construcción, la confección, la industria alimentaria y los servicios, entre otros) ${ }^{14}$. El modelo agroexportador imponía traslados campo-ciudad a los trabajadores y el pasaje por períodos de inactividad, lo que contradecía el presupuesto adoptado por el paradigma censal que le otorgaba al trabajador una posición fija en la estructura productiva.

En la Argentina del cambio de siglo, las situaciones de inestabilidad laboral o los períodos de inactividad eran habituales para los trabajadores ${ }^{15}$. Lejos de la percepción de los propios afectados que vivenciaban la falta de trabajo, la polémica intelectual se colocó en el plano de la propia necesidad de cuantificar el fenómeno de la desocupación; si la cuestión ameritaba o no la movilización de recursos del Estado. El desacuerdo era sintomático del resquebrajamiento del consenso liberal que había imperado en el país a fines del siglo XIX. La discusión ponía de manifiesto dos posiciones: por un lado, la de los liberales «clásicos» que suponían superfluo medir un fenómeno coyuntural de desequilibrio que el mercado mismo ajustaría, por sus propias leyes, automáticamente (posición encarnada por los estadígrafos de la comisión censal); por el otro, la posición del católico social Alejandro Bunge, compartida por los socialistas, que asumía que el mercado de trabajo no era un mercado como otros y que, por tanto, era necesaria cierta regulación por parte del Estado, como mínimo en lo que respecta a fenómenos disruptivos de la paz social. Las estadísticas sociales producidas en el DNT -como las de los bureau laborales de Francia, Alemania y Estados Unidos de fines de siglo XIX involucradas en la construcción del Estado providencia- partían

\footnotetext{
${ }^{14}$ Lobato, 2000.

15 Idem.
} 
de la concepción de que el mercado de trabajo debía estar en algún punto protegido. Al mismo tiempo, esas cifras actuaban como el principal elemento de justificación, tanto científica como política, de la ampliación de los márgenes de la política social del Estado.

Los primeros cuestionamientos a un modelo dominante de pensar las relaciones capital-trabajo en la Argentina no acarrearon, sin embargo, un consenso social suficiente sobre la naturaleza del problema de la desocupación y de los métodos necesarios para tratarlo. Desde el punto de vista de la agencia oficial, en 1913 el paro forzoso se presentaba como «la más importante y compleja de las cuestiones sociales. Castiga como una epidemia a toda una clase de individuos y afecta fundamentalmente a la sociedad entera» ${ }^{16}$. La falta de datos estadísticos abonaba, según Bunge, a visiones contrapuestas de la realidad del paro involuntario y sumía en la ignorancia a políticos e intelectuales preocupados por la cuestión: «¿Cómo podremos saber que en Buenos Aires falta trabajo si no hay cifras que nos digan el número de los desocupados que existe diariamente y la duración media de la desocupación? En ningún país del mundo se dio importancia al paro forzoso hasta que las cifras no descubrieron la gravedad del mal. (...) La estadística ha sido una asombrosa revelación aún para los más pesimistas $\rangle^{17}$. Para Bunge, sólo las estadísticas de la desocupación podían corroborar -o derrumbar una a una- las ideas previas sobre la desocupación. Como único criterio de verdad inobjetable, las cifras darían prueba de la existencia «real» de la situación, más allá de la percepción subjetiva de los propios afectados ${ }^{18}$.

Asimismo, la estadística constituía para Bunge el paso previo necesario para adoptar remedios adecuados y eficaces frente a una crisis del trabajo, puesto que permitirían implementar el seguro frente a la inactividad temporal o permanente. Sin una buena estadística del paro por profesiones no había seguro posible ${ }^{19}$. Como Bunge, sus continuadores en la DE-DNT comulgaron con la misma fe en la importancia de la estadística como base de cualquier legislación social que se pretendiera apropiada. Durante toda la década de 1920, sus propuestas y recomendaciones a los distintos poderes del Estado en

${ }^{16}$ Boletín del Departamento Nacional del Trabajo, febrero 1913: 6.

17 Ibidem: 40.

18 En julio de 1913, los socialistas presentaban en el periódico La Vanguardia cálculos, que decían oficiales, sobre la cantidad de obreros parados en la Capital Federal y planteaban que esos datos daban a la desocupación «las proporciones de una crisis de trabajo». El exceso inmigratorio era planteado como causa de la formación de ese ejército de reserva, por lo que consideraban necesario que los poderes públicos dejaran de fomentar «la inmigración artificial» como una forma de «previsión sana y patriótica» (La Vanguardia, 30/07/1913: 1).

19 Boletín del Departamento Nacional del Trabajo, febrero 1913: 41. 
materia laboral estuvieron dirigidas a regular las relaciones laborales y ampliar las protecciones sociales del trabajador y su familia ${ }^{20}$.

En 1913, Bunge cerraba con las siguientes palabras el primer capítulo de la discusión sobre la desocupación en la Argentina: «los datos precisos obtenidos demuestran que no se ha producido desocupación en la Capital, ni afluencia «anormal» de desocupados a ella» ${ }^{21}$. Las conclusiones a las que arribó Bunge tras su primer estudio sobre la desocupación urbana chocaron con la percepción general de los obreros, apoyados en su experiencia inmediata como principio de evidencia, y fueron públicamente cuestionados por sus organizaciones representativas ${ }^{22}$. La agregación estadística, sin embargo, había servido para que la cuestión de la desocupación empezara a ser construida como un problema de carácter colectivo (se identificara como un «mal» o una «enfermedad social» que afectaba a la sociedad en su conjunto) sobre el cual cabía la intervención del poder público. Si bien no se llegó a instrumentar un seguro de desempleo, como sí se hizo en Francia (1914) o Inglaterra (1911) tras la construcción estadística del problema de la desocupación, la emergencia de la representación del desocupado estuvo acompañada por la expansión de otro tipo de dispositivo institucional, la agencia estatal de colocaciones y el registro de los sin trabajo, lo que suponía una primera injerencia del Estado sobre el mercado laboral y cierto afán de coordinación de la oferta y demanda de trabajo. Según Topalov, los espacios de gestión de los trabajadores sin empleo fueron los ámbitos donde se propagó el vocabulario y las categorías creadas a fines del siglo XIX por reformadores y administrativos franceses y británicos para designar a los trabajadores sin trabajo, reorganizando las representaciones del trabajo y del no trabajo de los sectores populares ${ }^{23}$. En la

${ }^{20}$ Los especialistas del DNT intervinieron con cierta autoridad en la definición de la política laboral argentina. Según González Bollo (2004), la activa participación de los estadísticos del DNT se manifestó en la ley 10.505 de regulación del trabajo a domicilio (1918), los proyectos de asociaciones profesionales, el proyecto del código del trabajo del presidente Hipólito Yrigoyen (1921) y los proyectos de creación de consejos industriales mixtos (1920) y de junta económica nacional (1921 y 1923).

${ }_{21}$ Anuario Estadístico del Trabajo, 1913: 954.

${ }^{22}$ Los socialistas criticaron las conclusiones del «consumado teólogo de la ciencia aritmética», a quien denunciaban por realizar un manejo mal intencionado de las cifras públicas con la intención de querer demostrar que la desocupación no existía. El principio de evidencia de Bunge, fundado en la supuesta capacidad de la estadística de reflejar la realidad, chocaba con el de los socialistas que consideraban que la crisis del trabajo «aún se palpa» y continuaban exigiendo al poder político medidas (y mediciones), más allá de que «la intrincada red» de cifras del «estadígrafo clerical» dijera lo contrario. Al respecto, ver: «Los desocupados», $L a$ Vanguardia, 23/08/1913: 1; «La desocupación» (La Vanguardia, 13/12/1913: 1).

23 Topalov, 1994: 25. 
Argentina, en cambio, los límites de la categoría del desocupado continuarían estando difusos por un tiempo más, y el trabajador sin trabajo permanecería de alguna manera indiferenciado respecto de otras categorías, como las de los impedidos y los marginados.

\section{LOS LÍMITES BORROSOS DE UNA CATEGORÍA}

Orientada por Bunge, la DE-DNT se volcó a completar el vacío estadístico organizando un recuento de ocupados sobre los datos suministrados por los registros de talleres y fábricas. Lo que se hacía en la DE-DNT era comparar la cantidad de obreros registrada en dos momentos del tiempo y constatar un saldo negativo o positivo. Para Bunge, éste era un método indirecto válido para conocer el fenómeno de la desocupación, al menos en la ciudad Capital. Esta operación se repitió cada dos años. Aunque en la etapa posterior a la desvinculación de Bunge, la DE-DNT no desestimó otras fuentes como los registros de las agencias de colocaciones, promovió un censo fracasado en 1916 y una encuesta sobre la desocupación obrera en 1923, Bunge había dejado marcado el camino ${ }^{24}$.

Entre los intereses de la DE-DNT estaba observar «el movimiento constante de obreros que afluyen y refluyen, infundiendo energías vitales al gran mecanismo industrial de la Nación ${ }^{25}$. Para ello, era fundamental construir series temporales e inscribir la evolución de la desocupación en períodos de tiempo más largos. Entonces, los estadísticos laborales recurrieron a cálculos dudosos, apoyándose en censos previos, y realizaron estimaciones como la de «población obrera probablemente ocupada» ${ }^{26}$. Esta reconstrucción hacia atrás de los niveles de ocupación y desocupación era considerada importante en función de poder comparar años y diferenciar -como se venía haciendo en Estados Unidos desde 1886 con cada crisis- un nivel de desocupación «normal» de otro excepcional. El primero, era vinculado a una falta de trabajo habitual en la industria o a la inactividad temporaria, considerada una situación normal, mientras el segundo se lo asociaba a las depresiones económicas y

${ }^{24}$ El intento de censo de desocupados de 1916 se basó en la presentación espontánea de los desocupados para ser fichados en las comisarías seccionales. Posteriormente, el abogado José Figuerola, titular de la DE-DNT, calculó que la proporción que no se había presentado alcanzaba al $50 \%$ de los desocupados, afectando la confiabilidad de sus resultados, «un total de 10.073 que declararon carecer de ocupación» (Boletín del Departamento Nacional del Trabajo, 36, enero 1918: 12).

${ }^{25}$ Ibidem: 55.

${ }^{26}$ Ibidem: 11. 
al hecho de que estas coyunturas le hacían perder su trabajo a los obreros. El supuesto que subyacía era que la desocupación producto de la crisis no era de la misma naturaleza que la desocupación «normal». La diferencia entre desocupación «normal» y «anormal» fue el centro de las clasificaciones elaboradas por el ingeniero Lucien March (1859-1933) en la oficina laboral francesa a fines de siglo XIX, miembro de la Société de Statistique de París quien algo más tarde introdujo en Francia la estadística matemática inglesa. Para medir la evolución en el tiempo de la desocupación, Bunge aplicó el método de los números indicadores basándose en conceptos de economistas y estadísticos de formación matemática con gran reputación internacional, como Arthur Bowley (1869-1957), especialista inglés en estadísticas laborales cuyo perfil es el caso típico de un innovador técnico según la definición de Davidson $^{27}$. Habían pasado apenas seis años desde que Bowley utilizara los index numbers en las estadísticas del desempleo, cuando Bunge los introdujo al país en 1918 y, como el repique de una campana, se expandieron a todos los rincones de la estadística pública nacional. Como señaló el antropólogo Jorge Pantaleón, con esta adaptación Bunge «contribuyó decisivamente a la internacionalización del espacio social argentino. El país se convirtió en seriable, desde el punto de vista estadístico, bajo normas internacionales» ${ }^{28}$.

Pero, en los años 20, la cuestión controversial en torno a las formas de medir la desocupación seguía viva y se desenvolvió a la luz de los diferentes métodos utilizados en los países capitalistas avanzados, que venían desarrollando la estadística de la desocupación desde la última década del siglo XIX. Desde entonces, en el contexto internacional, tenía lugar un debate sobre la desocupación que tuvo como uno de sus principales escenarios la Conferencia Internacional de la Desocupación realizada en Paris, entre el 18 y el 21 de septiembre de 1910. Como resultado de esta reunión privada de especialistas en la temática, que contó con una delegación argentina, se fundó la Asociación Internacional por la Lucha contra la Desocupación que estuvo activa hasta la Primera Guerra Mundial ${ }^{29}$. Según Topalov, el período que enmarca las discusiones sobre la medición de la desocupación fue una época de intensa circulación internacional de ideas, experiencias y modelos ${ }^{30}$. La información

27 Davidson, 1990: 75. Arthur Bowley fue profesor de ciencias económicas y de estadística en la London School of Economics, y de matemática en University College, Reading. Fue presidente de la Royal Statistical Society. Junto a Udny Yule contribuyó a renovar los métodos de la estadística administrativa inglesa durante su actividad en el Board of Trade, entre 1902 y 1903. Su obra más importante fue Elements of Statistics (London, 1907).

${ }_{28}$ Pantaleón, 2009: 39.

29 Topalov, 1994: 60.

30 Ibidem: 273. 
se difundía rápidamente entre las administraciones estatales del trabajo que intercambiaban sus boletines. A su turno, los expertos argentinos de la DEDNT se sumaron a este circuito y ello les permitió estar empapados de los métodos aplicados en otros países, así como proyectar su propia labor en ese espacio trasnacionalizado. Los expertos locales conocían las experiencias de medición de la desocupación en otros países y, por tanto, entendían también en qué medida cada uno de ellos había mensurado, a través de sus métodos, cosas diversas ${ }^{31}$.

Sin embargo, ningún método fue totalmente desestimado por el bureau laboral. Los registros de las agencias de colocaciones o de sindicatos, que venían siendo utilizados en Estados Unidos, Francia e Inglaterra (aunque eran objeto de críticas), constituían en Argentina bases débiles donde apoyar la estadística de la desocupación. Frente a otros métodos como la encuesta o el operativo censal específico, esos mecanismos indirectos tenían la desventaja de excluir, en los hechos, a gran parte de la población, ya sea por que se basaban en la voluntad de registro de parte de los trabajadores sin empleo o porque no contemplaban a los trabajadores no sindicalizados que representaban a la mayoría de los obreros del país. Por otro lado, la administración de una encuesta resultaba dificultosa, por las mismas razones que un censo específico agravaba: la falta de recursos materiales y humanos de la agencia laboral argentina. Ante la falta de un consenso técnico respecto de la superioridad de una estrategia sobre otra, y siguiendo las recomendaciones internacionales, el DNT intentó aplicar todas las formas de recuento posibles, entendiéndolos como modos complementarios de medir el fenómeno de la desocupación.

Sin embargo, una cuestión fundamental subyacía a las diversas prácticas de recuento y tenía que ver con la definición misma de su objeto: el desocupado. Aún no existía entre el grupo de especialistas locales un consenso

${ }^{31}$ Los censos de Estados Unidos realizados entre 1880 y 1910, por ejemplo, incluyeron una pregunta relativa al desempleo. Esta pregunta figuraba en un cuestionario aparte, aun cuando el desocupado no se encontraba claramente definido, pues este problema no alcanzaba todavía demasiada relevancia política. La poca importancia brindada a esta distinción llevó incluso a que se suprimiera la pregunta en el censo de 1920. (Desrosières, 1996: 221) La aprehensión estadística del desocupado cobró otra relevancia con la profunda crisis social desatada en Estados Unidos tras el crack financiero de 1929. Cuestiones técnicas como a quiénes considerar desempleados, o cómo calcular la tasa de desempleados, raramente se habían planteado antes de 1930. La crisis económica y el contexto político, donde los demócratas le empezaban a reclamar al gobierno republicano una política nacional de lucha contra el desempleo, le dieron al tema otra relevancia pública. Sobre la experiencia norteamericana previa de clasificación de los desocupados a través de los censos de población, ver Topalov, 1994: 314-323. 
sólido respecto de qué contar, cuestión previa o complementaria al desafío de cómo contarlo. ¿Debía tratarse del número de jornales o días sin trabajo, de la cantidad de individuos afectados o de enumerar los casos de desocupación? Contar una u otra cosa traería resultados diversos e implicaría una forma de concebir y de encarar el problema del paro forzoso diferente. Bunge se inclinó por tomar como unidad de recuento a las personas, acorde con la estabilización que había logrado el término desocupación en Francia, donde de designar la ausencia de actividad en general (la de los domingos o feriados, por ejemplo) pasó a clasificar, en particular, a los obreros industriales asalariados modernos sin empleo.

En la Argentina, sin embargo, qué se entendía por desocupación quedaba aún pendiente de definición. La distinción entre desempleados, inactivos e invalidados para el trabajo permanecía difusa. La estrategia elegida por Bunge de comparar las planillas de ocupación obrera de las fábricas porteñas cada seis meses, o de un año a otro, para observar el saldo y establecer un cálculo aproximado de la desocupación, no discriminaba en realidad al interior de ese arco diverso de situaciones. La categoría estadística de desocupación, por su falta de precisión, terminaba equiparando la paralización de la actividad laboral por enfermedad, licencia o invalidez, e incluso por vejez, con las consecuencias negativas del funcionamiento del mercado de trabajo. Un debate taxonómico aún quedaba por saldarse que habilitara la distinción «real» $\mathrm{y}$ «en el papel» de los desocupados respecto de los inactivos o de los no aptos para el trabajo, pero también de la categoría de los marginados.

El problema cognitivo que se presentaba a la estadística pública remitía al de la constitución misma de la categoría de desocupado en el campo social. El desocupado era ubicado en cercanía con las diversas denominaciones de la pobreza marginal entonces en circulación, como mendigos, vagos o atorrantes. Incluso, podía llegar a ser asociado con el mundo del delito. La representación de la cercanía entre los mundos del trabajo y del delito había sido instalada y promovida por un discurso poderoso -en términos de su eficacia simbólica- como fue el de la criminología positivista que postuló la existencia de circuitos sociales donde el trabajador irregular o desocupado se convertía en el delincuente ocasional, más tarde profesionalizado ${ }^{32}$. La imagen de la proximidad de la situación de los desocupados temporarios o crónicos con la delincuencia parece haber permanecido durante largo tiempo en el imaginario social urbano. Esta representación, por ejemplo, estaba inmersa en las objeciones que presentó, en 1932, el Museo Social Argentino respecto de llevar adelante un censo de desocupados basado en la voluntad propia del individuo

32 Salvatore, 2000. 
de registrarse, ya que suponía que estos sujetos, racionalmente, tendrían la intención de escapar de la fiscalización del Estado ${ }^{33}$.

Ante las contaminaciones presentes entre categorías sociales, la estadística no alcanzaba a estabilizar e instaurar como indiscutibles nuevas clasificaciones. La dificultad del trabajo clasificatorio radicaba en separar aquello que no estaba del todo separado en la realidad social. ¿El mendigo, el vagabundo, el trabajador callejero, debía o no ser contado como desocupado? En su búsqueda por aprehender al desocupado, la DE-DNT reconoció la presencia de resistencias sociales a esa clasificación. La preocupación de los especialistas de la agencia laboral era que las personas negaran su condición de parados por el temor de que los tomaran «por vulgares vagabundos» ${ }^{34}$. Si bien la operación de clasificación de las personas pertenece al orden de lo cognitivo, la asignación de una identidad social suponía también una relación práctica entre clasificadores y clasificados. La pertinencia y el carácter operativo de la categoría del desocupado resultaron controversiales en la Argentina de entreguerras, como en otros casos nacionales.

La categoría de desocupado tampoco se encontraba desmarcada totalmente del grupo de población sujeta, real o potencialmente, a la asistencia del Estado. La tradición estadística nacional se diferenció de la de otros países de la región por una grilla censal que buscó capturar la población considerada como «no apta» o «incapaz», distinguiéndola del resto. En un registro que la comisión censal decimonónica denominó como «condiciones especiales» se agrupaba a las personas consideradas como «miembros pasivos o inútiles» de la sociedad, y que en ese sentido constituían -a los ojos de los censistas del siglo XIXuna carga social para el Estado ${ }^{35}$. El registro de los invalidados socialmente reapareció en el censo de la Capital Federal de 1936, que relevó el número de personas incapacitadas física o mentalmente para trabajar. En la pregunta formulada a los censados, la invalidez o enfermedad era puesta en relación directa con la imposibilidad de desempeñar un trabajo asalariado. Pero esta vez se trataba de reconocer a los no aptos para el trabajo y diferenciarlos de los desocupados, puesto que para los primeros se consideraba justificado su estado de inactividad. La capacidad para el trabajo separaba las aguas entre desocupados e invalidados, sumándose un elemento subjetivo a la definición

33 Boletín del Museo Social Argentino, 1932, octubre-diciembre: 318.

${ }^{34}$ Boletín del Departamento Nacional del Trabajo, 36, enero 1918: 53.

35 En el espacio dado por los tres primeros censos nacionales de población al registro de las «condiciones especiales» se mezclaban aspectos ligados a enfermedades (bocio), condiciones físicas (inválidos, ciegos, sordo-mudos) y psíquicas (dementes) con cuestiones relativas a la condición social (huérfanos e ilegítimos) de las personas, consideradas todas como elementos degenerativos del organismo social. 
de los primeros quienes pasaron a ser identificados, además, como quienes carecían de ocupación remunerada, pero tenían el deseo de trabajar.

Esta conceptualización cuenta como antecedente con las disposiciones de la Organización Internacional del Trabajo (OIT) a las que los estadísticos de la DE-DNT echaron mano en 1920 cuando, sobre un terreno de significados sociales polisémicos, la distinción precisa del desocupado como categoría estadística se tornaba dificultosa. El reconocimiento de la definición de la OIT implicó un primer paso en dirección a la autonomización de la categoría estadística del desocupado, pero no resultó concluyente. La oficina estadística de la OIT establecía que «el término desocupación no comprende: 1. A las personas desocupadas por razón de vejez, enfermedad o invalidez. 2. A las que no quieren o son incapaces de trabajar. [...] El concepto de desocupación sólo se extiende a las personas que quieren y pueden trabajar, pero que no trabajan porque, a pesar de sus esfuerzos, no encuentran ocupación ${ }^{36}$.

Al año siguiente de su creación (1919), el Departamento de Estadística de la OIT comenzó a establecer normas en materia de estadísticas relativas al mundo del trabajo. Sus tres primeras conferencias internacionales (1923, 1925 y 1927) tuvieron como objetivo universalizar la estadística de los hechos sociales relativos al trabajo, uniformizar formas de medición y presentación, y permitir comparaciones internacionales. El ámbito de aplicación de sus normas se limitaba a los países industrializados y a ciertos temas, entre los que figuraba el desempleo (junto a los salarios, el costo de la vida y los accidentes del trabajo, entre otros). Argentina era miembro de la OIT desde fines de junio de 1919. Sin embargo, el conocimiento de las normas estandarizadas a nivel internacional y de las recomendaciones técnicas de las conferencias de estadígrafos del trabajo no implicó, de parte de los especialistas locales, un acatamiento lineal a las resoluciones del organismo. Si bien estas resoluciones no eran de aplicación obligatoria para los países participantes, sino señalaban el punto de vista de los técnicos y tenían el valor de recomendaciones a los gobiernos, la DE-DNT hizo rutina un procedimiento de cálculo de los niveles de ocupación y desocupación que se desentendió de las precisiones que intentaba establecer la OIT.

Los estadísticos de la DE-DNT se integraron al circuito internacional de producción y difusión de estadísticas laborales, a pocos años de creado, aunque no claramente desde una posición central; mantenían un intercambio con sus pares de otras oficinas del mundo y accedían a las publicaciones más importantes en la materia, como la Revue Internationale du Travail, órgano de la OIT donde fueron publicadas las estadísticas internacionales del desempleo

\footnotetext{
${ }^{36}$ Boletín del Departamento Nacional del Trabajo, 45, febrero 1920: 97.
} 
desde 1921. En el marco del movimiento de estandarización internacional de conceptos y mediciones impulsado por el organismo, Alejandro M. Unsain, un funcionario calificado y de larga trayectoria en la oficina laboral local (que alcanzó incluso a ser presidente interino del DNT), participó en 1927 de la Tercera Conferencia de Estadística del Trabajo de la que surgieron recomendaciones técnicas respecto de los métodos para recoger y publicar, bajo un formato uniforme y por períodos de tiempo homologables, estadísticas sobre la desocupación. En aquella ocasión, representando al país, Unsain se puso en contacto con los jefes de los servicios de estadística del trabajo de las principales naciones de Europa. Allí, por ejemplo, el especialista laboral argentino se relacionó con Conrado Gini (1884-1965), reconocido estadístico, demógrafo y sociólogo que fuera director del servicio estadístico de Italia. Los lazos rindieron sus frutos puesto que pocos años después hubo otra delegación argentina en el Congreso Internacional de Población organizado por Gini en Roma en 1931. Más tarde, Gini fue nombrado miembro honorario de la Sociedad Argentina de Estadística, fundada en 1937, que tuvo como vicepresidente al jefe de la oficina de la estadística laboral, José Figuerola.

Este abogado español fue nombrado jefe de la DE-DNT en 1931 por Eduardo F. Maglione, presidente de la agencia laboral durante la presidencia del General Uriburu. En el transcurso de su gestión (hasta 1943), la repartición estadística amplió sus investigaciones, incorporó oficinas provinciales ampliando su alcance geográfico, aceleró el ritmo de actualización de los datos y diversificó sus publicaciones. En 1941, contaba con un plantel de 24 empleados (que más que duplicaba al de la década de 1920). El decreto 50.720 de octubre de 1934 le otorgó a la DE-DNT plena autonomía técnica para determinar criterios y procedimientos de medición de los fenómenos laborales y sobre definiciones operativas como cuándo levantar los censos. A partir de los años 1930, esta agencia burocrática especializada era considerada en el país como un laboratorio social moderno. En el marco de una Nación en proceso de industrialización, los números del DNT se habían transformado en los códigos socialmente válidos de lectura del mundo del trabajo ${ }^{37}$. En 1932, Figuerola dirigía el primer Censo Nacional de Desocupados que se realizaba en la Argentina, basado en la presentación voluntaria en las oficinas públicas del Registro Nacional de Colocaciones de quienes se auto-identificaban como tales.

${ }^{37}$ Figuerola fue Secretario General de la Secretaría de Trabajo y Previsión (1943-1944), luego cumplió esa misma función en el Consejo Nacional de Posguerra (1944-1946) y más tarde ascendió a la Secretaría Técnica de la Presidencia de la Nación (1946-1949) en el primer gobierno de Juan Domingo Perón. Para profundizar en su trayectoria ver González Bollo, 2011. 


\section{EL FRACASO DE LA ENUMERACIÓN EXHAUSTIVA}

Hacia 1930, la situación de crisis internacional que había afectado los niveles de empleo de los países capitalistas avanzados fue el marco en el que se terminó de consolidar en la Argentina el tema de la desocupación como cuestión social, a espejo de lo que acontecía en aquellas naciones. La circulación local de estadísticas sobre la situación del paro obrero en otras latitudes instaló la pregunta respecto de cómo se manifestaba el problema en la Argentina. Si bien en los años inmediatamente previos había sido desplazada de los primeros planos de la agenda política, la cuestión de la desocupación venía resonando en el campo académico-intelectual desde hacía tiempo: era un tema abordado en congresos (como el Congreso Internacional de Economía Social, promovido por el MSA en octubre de 1924, en el que Bunge presidió la mesa sobre estadística social y cuestiones sociales), se había convertido en materia de programas de estudio (la carrera de servicio social creada por el MSA en 1929 contaba con una materia de primer año denominada pauperismo y paro forzoso) y circulaba en revistas especializadas, como la de la FCE-UBA, que llevaron adelante encuestas de opinión respecto al tema de la desocupación a personas reconocidas públicamente como especialistas.

En 1928, la Comisión de Legislación del Trabajo de la Cámara de Diputados le exigió al DNT la elaboración urgente de información sobre ocupación y desocupación obrera en la Capital Federal. La oficina laboral se excusaba de la imposibilidad de satisfacer esta demanda aduciendo que en el país no estaban dadas las condiciones para tener esos datos, ya que no existían las bolsas de trabajo de las asociaciones gremiales ni las cajas de seguro frente al paro, fuentes tradicionales de la estadística del desempleo en los países pioneros en la materia. La estadística local continuaba mirando los modelos de Francia e Inglaterra, que se apoyaban en fuentes administrativas o de las propias organizaciones de trabajadores, y se lamentaban de la distancia que la separaba de estos bureau laborales, mientras en Estados Unidos se comenzaba a aplicar una encuesta por muestreo para establecer la cifra oficial del desempleo ${ }^{38}$. Los cuadros de la estadística laboral argentina seguían pensando en términos de enumeración exhaustiva, mientras que la posibilidad de tomar a la parte por el todo, a partir de la selección aleatoria de una muestra -tal como había sido propuesto por el estadígrafo noruego

${ }^{38}$ Para profundizar en la historia de la aplicación del método de la encuesta por muestreo en la medición del desempleo, ver Desrosières, 1996: 219-230. Para los comienzos de la noción de representatividad y las discusiones en la comunidad estadística internacional, Desrosières, 1996: 246-254. 
Anders Kiaer a fines de siglo XIX-, lograba al fin su coronación en los Estados Unidos de los críticos años ' 30 con la aplicación de la técnica de la encuesta por sondeo para medir el desempleo. Esta herramienta estadística estaría ligada, a su vez, a la instauración del dispositivo de indemnización por desempleo en 1937.

Sin embargo, en la Argentina, la noción de representatividad tardaría bastante más en popularizarse ampliamente. Desde el punto de vista de Figuerola, para asentar una afirmación matemáticamente exacta respecto de los alcances de la desocupación era necesario un censo auténtico de los obreros, empleados, dependientes y aprendices ocupados en todo el país y, además, un riguroso padrón de habitantes. La ausencia de tales instrumentos hacía imposible, en su opinión, acceder a conclusiones firmes respecto del paro involuntario y formular explicaciones científicas en las que establecer las bases de las políticas sociales ${ }^{39}$.

En 1932, el Congreso Nacional designó los fondos para realizar el primer Censo Nacional de Desocupados (ley 11.590). ¿Qué había cambiado entre la crisis de 1913/17 y la de 1930 para esta vez sí lograr que el Estado desplegara (y con urgencia) un operativo nacional de medición de la desocupación? Las depresiones económicas y las crisis de desocupación (repentinas y masivas) habían pasado a ser visualizadas como situaciones perturbadoras de un orden en el que ya no era más «natural» que el obrero, por ciertos momentos, se encontrara sin trabajo. Si bien la interrupción de la actividad laboral (su irregularidad e inestabilidad) era característica del mercado de trabajo argentino para 1914 y formaba parte de la experiencia obrera habitual, el posterior proceso de desarrollo industrial experimentado por el país había estado acompañado por una mayor regularidad del empleo y por el desarrollo de una legislación laboral, si bien parcial o que regulaba sólo determinados aspectos de la relación laboral en ciertos sectores de actividad, significó los primeros avances en dirección a la institucionalización del estatuto del asalariado. La expansión industrial era un factor clave en la medida en que la configuración de la desocupación como problema social y estadístico tuvo siempre en la Argentina como objeto al trabajo urbano, antes que al rural. La estabilización del empleo, la industrialización del país y los avances en materia de legislación social, contribuyeron a configurar una crisis del trabajo como una situación anormal,

39 Departamento Nacional del Trabajo, 1933: 150. Aun cuando, después de reiteradas experiencias, el método del censo por registro voluntario demostró ser un fracaso, Figuerola no propuso para su reemplazo una investigación por muestreo, sino la actualización permanente del censo profesional que, mediante el registro de las altas y las bajas del personal ocupado, le brindaría a la DE-DNT un procedimiento indirecto para estimar el desempleo involuntario. 
excepcional, que reclamaba medidas urgentes apoyadas en un fundamento racional: las estadísticas de los parados.

La realización de un Censo Nacional de Desocupados fue propuesta por una comisión del Congreso creada para combatir la desocupación y asesorada por el DNT ${ }^{40}$. En la sesión en la que se trató el proyecto, el diputado socialista por la Capital Federal Nicolás Repetto, integrante de la comisión, se refirió a la falta de información del Estado y criticó el carácter exclusivamente porteño de la estadística producida por la oficina laboral que ignoraba cuáles eran las regiones del país más azotadas por la desocupación ${ }^{41}$. Estaba claro que la desocupación había dejado de ser un problema local y se había constituido firmemente como un hecho social que exigía una medición abarcadora de toda la Nación. El interés político recaía, fundamentalmente, en establecer la distribución geográfica de la desocupación porque lo que se reclamaba al Estado era una política integral de lucha contra el desempleo.

En esta oportunidad, la definición del procedimiento técnico que debía llevarse a cabo no concitó ninguna polémica; se haría un censo especial basado en la presentación voluntaria de los parados. El debate abierto en la sesión parlamentaria del 24 de junio de 1932 giró en torno al contenido de la fícha censal. La discusión remitía a los principios legítimos de percepción oficial del fenómeno de la desocupación que serían representados por las preguntas a hacerle a los censados. Algunos diputados no consideraban conveniente que el censo indagara en demasiados detalles, temiendo que ello elevara sus costos financieros y retrasara la presentación de sus resultados. Desde su punto de vista, la urgencia por instrumentar el censo de desocupados exigía aligerar la cantidad de datos a recabar. Sin embargo, otros diputados consideraron que el censo no se podía estrechar tanto como para perder utilidad como fuente racional de la política social. El punto central de la discusión abierta en el Congreso fue la necesidad de indagar si los desocupados eran nacionales o extranjeros. Para algunos diputados, la nacionalidad de los parados, y su tiempo de permanencia en el país, eran datos esenciales.

En este sentido, el representante del partido demócrata nacional de Mendoza, Adolfo Vicchi, argumentaba que era importante conocer el lugar de residencia y la nacionalidad de los desocupados porque era la forma de corroborar si el hecho social se producía en el país como consecuencia de una excesiva inmigración de desocupados. Su copartidario, el diputado por Entre Ríos Francisco Maglione, lo consideraba un dato fundamental para el diseño

${ }^{40}$ La comisión del Congreso estaba conformada por los senadores Patrón Costas, Bravo, Santamarina y los diputados Repetto, Sierra, Parera, Biancafiore y Costa Méndez.

41 Departamento Nacional del Trabajo, 1933: 153. 
de la legislación «porque si bien el Estado, por humanidad, debe procurar trabajo a todos los que lo piden, debe empezar por proporcionárselo a los criollos, a los hijos del país, antes que a los extranjeros» ${ }^{42}$.

Mientras la crisis, como telón de fondo que se combinaba con el resurgimiento de la derecha nacionalista, precipitaba el trabajo clasificatorio, en la discusión en el Congreso se forjaban las categorías de representación necesarias para la formulación de políticas públicas ${ }^{43}$. Incorporar o no una pregunta al censo se convertía en mucho más que una definición técnica: implicaba asumir un presupuesto explicativo del fenómeno (la considerada excesiva afluencia extranjera al mercado de trabajo local) y fundar, a la vez, una política selectiva sobre la materia en función del origen nacional del desocupado. La discriminación entre nacionales y extranjeros se erigía como principio organizador tanto cognitivo como político.

Finalizada la discusión, la distinción entre nativos e inmigrantes no sólo resultó avalada en el Congreso (las preguntas por la nacionalidad y el tiempo de residencia en el país formaron parte del cuestionario) sino que, una vez levantado el censo, se convirtió en el eje de la interpretación del problema sostenida por la Junta Nacional contra la Desocupación ${ }^{44}$. La Confederación General del Trabajo, que había formado parte de esta comisión, consideró que la lectura que se hacía de las cifras arrojadas por el censo era sesgada y abrió una nueva controversia pública tildando a la Junta de fascista. La entidad obrera tomó esas mismas estadísticas para contradecir sus explicaciones y oponerse a las medidas auspiciadas por la Junta.

Está claro que los resultados del primer censo de desocupados de 1932 no lograron una amplia credibilidad social. Los diputados socialistas desconfiaron públicamente de la cantidad final de desocupados que representaba el 2,4\% de la población total del país. Sus defensores esgrimieron que, a la luz de esas cifras, el problema del paro involuntario no era grave y que la cantidad de parados estaba asociada al momento del año en que se había realizado el operativo (en relación al ciclo productivo del agro). El censo fue respaldado por el Poder Ejecutivo en la voz de su Ministro del Interior, Leopoldo Me-

42 Ibidem: 154.

${ }^{43}$ Las propuestas de medidas para que el problema del paro obrero fuera administrado por el Estado de manera centralizada que circulaban entonces eran la construcción de obras públicas, una baja del salario mínimo para contener cesantías, colocar a desocupados, repatriar extranjeros que así lo desearan (pasajes gratis), e instrumentar el traslado voluntario de los desocupados al interior del país.

${ }^{44}$ La Junta Nacional de la Desocupación estaba conformada por entidades representativas de intereses sectoriales como la Sociedad Rural Argentina, la Bolsa de Comercio, la Unión Industrial Argentina y la Confederación General del Trabajo. 
$10^{45}$. Pero, en un informe oficial, el experto laboral José Figuerola admitió las limitaciones del operativo y puso aun más en duda su credibilidad al manifestar que no era posible tomar a los desocupados inscriptos en ese censo como expresión de la desocupación «real» ${ }^{46}$.

El censo de 1932 no terminó de despegarse del enfoque tradicional sobre el trabajo que había imperado en los censos argentinos de población; no incorporaba la distinción entre la población económicamente activa e inactiva, presente en la estadística norteamericana entonces faro de la estadística internacional, e introducida, por ejemplo, en el censo chileno de 1930. El propósito del censo había sido el de establecer la extensión del fenómeno de la desocupación en el país y conocer las características de la situación de los parados. Y es en este orden de cosas que surge el aporte novedoso del primer censo nacional de desocupados. En su configuración estadística, a través del censo los desocupados fueron clasificados en cuatro categorías que pretendían plasmar la distinta naturaleza del paro que los afectaba: 1. los desocupados totales y permanente; 2. los desocupados totales y circunstanciales; 3 . los desocupados parciales y, por último, 4. los desocupados periódicos o de temporada ${ }^{47}$.

La lógica principal de la clasificación no era tanto la exclusión (quien quedaba dentro o fuera de la categoría de desocupado) como el recubrimiento parcial, acumulativo, de los matices de un fenómeno social complejo que se pensaba heterogéneo a su interior. Asimismo, la clasificación se estructuraba sobre la base de dos pares de términos opuestos: parcial/total y circunstancial/ permanente. Entre el desocupado total y el parcial se dirimían dos formas de concebir la desocupación: la idea de la desocupación como un estado (de carencia de una ocupación remunerada) versus la desocupación como un punto de pasaje, un momento particular en el ejercicio de una actividad, oficio o profesión habitual que se caracterizaba por su irregularidad. La diferenciación entre desocupados circunstanciales y permanentes distinguía, al interior de un grupo social estable, las personas que habían caído en ese estado -la falta de

${ }^{45}$ Memoria del Ministerio del Interior, 1932: 404.

46 Departamento Nacional del Trabajo, 1933: 150.

47 Según las definiciones publicadas oficialmente en la obra La Desocupación en la Argentina, 1932 (1933): 1) los desocupados totales y permanentes comprendían a los que con anterioridad al $1^{\circ}$ de enero de 1932 ya no tenían ocupación lucrativa; 2) los desocupados totales y circunstanciales involucraban a los que habiendo tenido trabajo regular antes del $1^{\circ}$ de enero de 1932 carecían de él a partir de esa fecha; 3) los desocupados parciales comprendían a los que a pesar de tener ocupación fija, sólo trabajan algunos días a la semana, o sin tenerla, realizan algún trabajo eventual con más o menos regularidad; 4) la categoría de los desocupados periódicos o de temporada comprendía a los que habitualmente se ocupaban de trabajos tales como la recolección de cosechas, y que luego no efectuaban ningún otro trabajo lucrativo. 
trabajo- si lo habían hecho hacía largo tiempo o de manera reciente. En la categoría de desocupados totales quedaron identificados las tres cuartas partes de los desocupados que se registraron voluntariamente. En cambio, para los desocupados parciales y periódicos o de temporada el no trabajo era una situación elegida o aceptada, que venía añadida al tipo de tarea o actividad que se realizaba, como en gran parte de los trabajos rurales.

El propósito general de la clasificación fue el de distinguir las distintas razones de la falta de empleo y las categorías de individuo que le correspondían. En los cuadros de presentación de la información, los desocupados fueron discriminados entre varón y mujer -por su peso relativo en el mercado laboral-, según su nacionalidad - retomando el punto tan controversial- y en función del oficio, especialidad o actividad profesional habitual de los parados, para conocer los sectores de actividad o, en términos de la época, las industrias más afectadas. A cada categoría distinguida por el análisis le correspondería un modo particular de tratamiento, la aplicación de ciertas medidas políticas y la puesta en práctica de dispositivos institucionales específicos. La idea era desarrollar los remedios adaptados a cada circunstancia. Por tanto, más allá de una simple operación estadística, la clasificación de la población desocupada constituía una operación estratégica. La forma en que se pensaba el problema de la desocupación y las posibles soluciones no había sido tanto el resultado del censo como el principio inicial de la clasificación, a partir de la cual los datos fueron organizados y portaron su inteligibilidad. Por tanto, definir problemas, establecer las causas, clasificar y contar a la población, prescribir soluciones, son términos inseparables de un mismo proceso.

\section{A MODO DE EPÍLOGO: LA INSTITUCIONALIZACIÓN DE UNA PRÁCTICA}

De 1932 en adelante, la DE-DNT buscó hacer el seguimiento de las oscilaciones del paro involuntario con censos periódicos de desocupados. Entonces, resultaba significativo conocer el nivel general de la desocupación y cómo se producía, en cada sector de actividad, el avance o el retroceso del paro involuntario, por considerarlo el síntoma característico del flujo y reflujo del movimiento económico-social ${ }^{48}$. Ya no se trataba, como en 1914, de llegar a la simple expresión numérica de un total de desocupados -mera sumatoria o agregación de individuos- sino de conocer el volumen de la desocupación -lo que constituía a la desocupación en un hecho social además de un objeto estadístico- e inscribirlo en el devenir de la sociedad, a través de índices y

\footnotetext{
${ }^{48}$ Revista Investigaciones Sociales, 1939: 44.
} 
series cronológicas. En 1934, el diputado socialista por la Capital Federal Rómulo Bogliolo afirmaba en la sesión del Congreso del 27 de junio: «lo que deseamos es que se comprenda de una vez por todas que la desocupación no es el problema surgido de la mera reunión de mil o dos mil hombres en Puerto Nuevo o en cualquier lugar deshabitado. Se trata de saber cuántos hombres arraigados en el país y con familia, de la noche a la mañana, no encuentran la manera de ganar el sustento para ellos y los suyos ${ }^{49}$. Entre 1918 y 1932, aquello que era objeto de medición estadística se había visto transformado.

En 1934, la sanción de la ley 11.868 dispuso el levantamiento de censos de desocupados cada seis meses, pero sólo se realizaron otros tres (en 1935, 1936 y 1940) con resultados poco satisfactorios. En ellos se expresaban, según Figuerola, cifras «incongruentes y dispares» que no podían ser consideradas «verdadera expresión de la realidad $»^{50}$. El sistema de censos periódicos había resultado inconducente, en opinión del especialista, porque era incapaz de capturar los «desocupados invisibles», es decir, quienes no habían tenido voluntad de inscribirse en el registro; situación no desestimable si se toma en cuenta la acusación del diputado Bogliolo respecto de los fines policiales que estaban implícitos en el censo de desocupados y la manera en que la institución policial tuvo participación en las tareas censales ${ }^{51}$.

Según la historiadora Mirta Lobato, con la transformación del DNT en Secretaría de Trabajo y Previsión, el organismo «pierde dinamismo en el plano cognitivo ${ }^{52}$ para enfocarse, por un lado, en la gestión de las relaciones directas de Perón con los sindicatos, las que le permitieron acumular poder político, $\mathrm{y}$, por el otro, en la aplicación de las leyes que promovieron el bienestar de los trabajadores. Con la introducción de la idea de planificación en la gestión de gobierno, las estadísticas ganarían más y más terreno como instrumento de conocimiento social, desde los primeros planes quinquenales a los posteriores programas nacionales de desarrollo. La medición de la desocupación se institucionalizaría entonces con la Encuesta de Empleo y Desempleo (EEyD), realizada por primera vez en el mes de julio de 1963 y transformada en un programa de relevamiento periódico por el Consejo Nacional de Desarrollo (CONADE), en articulación con el Ministerio de Trabajo y Seguridad Social. La iniciativa buscaba generar los indicadores sociales que tradujeran al lenguaje estadístico aquello que era juzgado socialmente como problema y que, por tanto, era objeto de intervención pública: el mercado de trabajo. En

\footnotetext{
49 Diario de Sesiones de la Cámara de Diputados, 1934, tomo 2: 812.

50 Revista Investigaciones Sociales, 1939: 43.

${ }^{51}$ Diario de Sesiones de la Cámara de Diputados, 1934, tomo 2: 811.

${ }^{52}$ Lobato, 2010: 109.
} 
correspondencia con el lugar central del trabajo en la agenda de las cuestiones sociales, el desocupado alcanzaría entonces una definición estadística estable; al mismo tiempo, la pretensión de recuento exhaustivo quedaría definitivamente desplazada por el método de encuesta en hogares sobre la base del muestreo aleatorio. El dispositivo de medición de la desocupación se sostendría, a partir de ahora, no sólo en herramientas más aggiornadas a los tiempos mundiales, sino en alianzas con otros actores sociales de creciente protagonismo y prestigio social en la década de 1960, ligados a las nuevas instituciones tecnocráticas creadas por el desarrollismo y al campo académico ${ }^{53}$.

\section{BIBLIOGRAFÍA}

Castel, Robert, La metamorfosis de la cuestión social, Buenos Aires, Paidós, 1997.

Daniel, Claudia, La sociedad (des)cifrada. Configuraciones del discurso estadístico en Argentina (1890-1945), Tesis de doctorado, Universidad de Buenos Aires, 2010.

Daniel, Claudia, «Una escuela científica en el Estado. Los estadísticos oficiales en la Argentina de entreguerras», Mariano Plotkin y Eduardo Zimmermann (comp.), Saberes de Estado, Buenos Aires, Edhasa, 2012: 63-101.

Davidson, Roger, «La formation du Labour Department. Le département du travail et la question ouvrière en Grande-Bretagne de la fin de la période victorienne à la période édouardienne», Genèses, 2 (1990): 56-82.

Davidson, Roger, «The state and social investigation in Britain, 1880-1914», M. J. Lacey y M. O. Furner (eds.), The state and social investigation in Britain and the United States, Cambridge, Cambridge University Press, 2004: 242-275.

Departamento Nacional del Trabajo, «La Desocupación en la Argentina, 1932», Informe del Jefe del Censo Nacional de Desocupados, Dr. J. Figuerola, Buenos Aires, Talleres Gráficos Compañía Impresora Argentina S. A., 1933.

Desrosières, Alain, La política de los grandes números. Historia de la razón estadística, Barcelona, Melusina, 1996.

Desrosières, Alain, «Las palabras y los números. Para una sociología de la argumentación estadística», apuntes de investigación del CECYP, (enero - junio 2011): 75-101.

53 Nos referimos a la vinculación de la EEyD con centros de saber técnico especializado como el CONADE, por un lado, y la Universidad de Buenos Aires, por el otro, representada en los vínculos iniciales de la EEyD con el Instituto de Sociología de la Facultad de Filosofía y Letras y, después, por medio de la figura del ingeniero Sigfrido Mazza, profesor de la Facultad de Ingeniería de la UBA y del Instituto de Cálculo de la Facultad de Ciencias Exactas y Naturales de la UBA, encargado de la muestra y los métodos de estimación de la EEyD. 
Gerchunoff, Pablo y Llach, Lucas, El ciclo de la ilusión y el desencanto: un siglo de políticas económicas argentinas, Buenos Aires, Ariel, 1998.

González Bollo, Hernán, «Ciencias sociales y sociografía estatal. Tras el estudio de la familia obrera porteña, 1899-1932», Estudios Sociales, 16 (Santa Fe, 1999): 19-39.

González Bollo, Hernán, «La cuestión obrera en números: la estadística socio-laboral y su impacto en la política y la sociedad, 1895-1943», Hernán Otero (dir.), El mosaico argentino. Modelos y representaciones del espacio y de la población, siglos XIX-XX, Buenos Aires, Siglo XXI, 2004: 331-375.

González Bollo, Hernán, «José Francisco Figuerola: de funcionario del estado interventor conservador a experto de la coalición peronista (1930-44)», <http://historiapolitica.com/datos/biblioteca/gbollo2.pdf>, consultado el 16 de marzo de 2011.

Lobato, Mirta Z., «Historia de las instituciones laborales en Argentina: una asignatura pendiente», Revista de Trabajo, año 3, no 4 (Buenos Aires, 2007): 145-154.

Lobato, Mirta Z., «Instituciones laborales, funcionarios y política: notas a partir de un proyecto de investigación», Revista de Trabajo, año 6, $\mathrm{n}^{\circ} 8$ (Buenos Aires, 2010): 105-116.

Lvovich, Daniel y Suriano, Juan, Las politicas sociales en perspectiva histórica, Argentina 1870-1952, Buenos Aires, Universidad Nacional de General Sarmiento/ Prometeo Libros, 2006.

Otero, Hernán, Estadística y Nación. Una historia conceptual del pensamiento censal de la Argentina moderna, 1869-1914, Buenos Aires, Prometeo editorial, 2006.

Panettieri, José (comp.), Argentina: trabajadores entre dos guerras, Buenos Aires Eudeba, 2000.

Pantaleón, Jorge, Una nación a medida, Buenos Aires, Ediciones Al Margen, 2009.

Rueschemeyer, D. \& Skocpol, T. (eds.), States, Social Knowledge, and the Origins of Modern Social Policies, New York, Princeton University Press, 1996.

Salvatore, Ricardo, «Criminología positivista, reforma de prisiones y la cuestión social/obrera en Argentina». Juan Suriano (comp.), La cuestión social en la Argentina, 1870-1943, Buenos Aires, La Colmena, 2000.

Suriano, Juan (comp.), La cuestión social en la Argentina, 1870-1943, Buenos Aires: La Colmena, 2000.

Suriano, Juan, «El Departamento Nacional del Trabajo y la política laboral durante el primer gobierno de Hipólito Yrigoyen». Mariano Plotkin y Eduardo Zimmermann (comp.), Saberes de Estado, Buenos Aires, Edhasa, 2012: 35-62.

Tercer Censo Nacional, levantado el 1 de junio de 1914. Buenos Aires: Talleres Gráficos de L.J. Rosso y Cía., 1916.

Topalov, Christian, Naissance du chômeur. 1880-1910, Paris, Albin Michel, 1994. 
Torrado, Susana, Población y bienestar en la Argentina del primero al segundo centenario, Buenos Aires, Edhasa, Serie Estudios del Bicentenario, 2007.

Zimmermann, Eduardo, Los liberales reformistas. La cuestión social en la Argentina, 1890-1916, Buenos Aires Sudamericana, 1995.

\section{FUENTES DOCUMENTALES}

Anuario Estadístico del Trabajo.

Boletín del Departamento Nacional del Trabajo.

Boletín del Museo Social Argentino.

Crónicas del Departamento Nacional del Trabajo.

Investigaciones Sociales.

La Vanguardia (periódico).

Memorias del Ministerio del Interior de la República Argentina.

Fecha de recepción: 9/11/12

Fecha de aceptación: 17/1/13

From crisis to crisis: the invention of unemployment in Argentina.

The article examines the historical process of configuration of unemployment as a social and statistical problem in Argentina during the interwar period, articulating three axes or dimensions: the link between statistical tools and forms of State, the international flow of knowledge around the issue of work, the role of crises (1914-1917 and 1930) in the production of stable statistical representations about unemployment in the country.

KeY-words: Unemployment; Social statistics; National Labour Department; Crisis; Argentina; Interwar period. 